\title{
A 7-year surveillance of the drug resistance in Klebsiella pneumoniae from a primary health care center
}

\author{
Guogang Li', Sheng Zhao', Sipei Wang ${ }^{1}$, Yingqian Sun ${ }^{1}$, Yangxiao Zhou ${ }^{1}$ and Xinling Pan²*
}

\begin{abstract}
Background: The increased prevalence of Klebsiella pneumoniae infections and resistance rates are a current cause for concern. However, data for resistance rates in K. pneumoniae strains from primary hospitals and the resistance distribution among the different isolate sample sources are scarce.

Methods: All the K. pneumoniae strains were isolated from patients who visited a primary health care center located in Central Zhejiang Province from January 2011 to December 2017. The specimens included blood, sputum, cervical secretions and urine. The species were identified by the Vitek 2 Compact Bacterial Identification and Monitoring System or VITEK-MS and the extended spectrum $\beta$-lactamase (ESBL) and drug resistance profiles were identified using the AST-GN13 Gram negative susceptibility card (VITEK-2). The genotype of strains from urine sources was analyzed by detecting TEM and SHV genes. Finally, the drug resistance rates among the isolates from different sample sources were analyzed using the Chi square test with SPSS software.
\end{abstract}

Results: A total of 5319 K. pneumoniae strains were isolated in this study. Among the 20 antimicrobial drugs studied, the resistance rates of $K$. pneumoniae strains varied from 1.4\% (ertapenem) to $23.1 \%$ (nitrofurantoin). The antibiotic resistance rates varied significantly among the isolate samples sources for all, with the highest rates for all antibiotics except for nitrofurantoin found in urine samples. In addition, the ESBL-positive rate in urine samples was 27.1\%, significantly higher than that of cervical secretions (20.2\%), blood (16.5\%) and sputum (15.2\%). Compared to the ESBL-negative strains, higher resistance rates were detected in the ESBL-positive strains. The most common genotype of isolates from urine was SHV (28\%, 23/82), following by TEM (14.6\%, 12/82).

Conclusion: The highest resistance rates of $K$. pneumoniae strains to most antibiotics found in urine samples are partly due to the ESBLs, indicating that a special attention should be paid in the treatment of urinary tract infection.

Keywords: Klebsiella pneumoniae, Drug resistance, Urine, ESBL

\section{Background}

As a common opportunistic pathogen, Klebsiella pneumonia (K. pneumoniae) causes a wide range of infectious diseases including, urinary tract and soft tissue infections, bacteremia, and pneumonia. It is also an important causative agent of serious community-onset infections,

\footnotetext{
*Correspondence: panfengyuwuzu@163.com

${ }^{2}$ Department of Biomedical Sciences Laboratory, Affiliated Dongyang Hospital of Wenzhou Medical University, No. 60 Wuningxi Road, Dongyang, Zhejiang, China

Full list of author information is available at the end of the article
}

including pyogenic liver abscess and necrotizing pneumonia $[1,2]$.

As a result of the abuse and misuse of antimicrobial drugs, resistance to these agents is emerging as a worldwide crisis in the treatment of $K$. pneumoniaeassociated infections [3]. A study of 3,132,354 antimicrobial susceptibility results from the United State revealed significantly increased drug resistance rates for all agents except tetracycline during the period from 1998 to 2010 [4]. Moreover, the increasing resistance of clinical isolates to the carbapenems (imipenem) and the fourth-generation cephalosporin cefepime, which 
are traditionally considered to be most effective agents against $K$. pneumoniae, is a major cause for concern $[4,5]$. Clonal expansion of drug-resistant strains has resulted in outbreaks in hospitals in several countries [6], leading to a high mortality in infected individuals.

The exact mechanism underlying drug resistance in $K$. pneumoniae strains remains to be clarified, although the extended-spectrum $\beta$-lactamases (ESBL) have been reported to play an important role [7]. ESBLs in $K$. pneumoniae often confer resistance to advanced generation cephalosporins, leading to the therapeutic failure of these agents. In addition, ESBL-associated plasmids often carry genes encoding co-resistance to other antibiotics, resulting in multi-drug resistance phenotypes [8].

Investigations of the prevalence of drug-resistant strains in clinical hospitals are urgently required to determine optimal treatment strategies in patients with $K$. pneumoniae infections. However, most of the data about the prevalence and drug resistance of K. pneumoniae in China were obtained in large city hospitals, where the patients are more likely to have severe infections $[9,10]$. Moreover, most of the studies focused on the strains causing infection in a specific cohort [11, 12] (such as infants, the elderly or intensive care unit (ICU) patients) or infection site (such as blood and liver) [13, 14], while others have investigated the molecular epidemiology of the hypervirulent strains [15].

However, the global distribution and drug resistance of $K$. pneumoniae strains among the clinical samples and the contribution of ESBL to drug resistance remain to be elucidated. Therefore, we conducted a retrospective analysis of the $K$. pneumoniae strains isolated in a primary hospital located in southeastern China during the period from 2011 to 2017.

\section{Materials and methods Clinical isolate collection}

The strains were isolated from patients (outpatients and inpatients) who visited the Affiliated Dongyang Hospital of Wenzhou Medical University from January 2011 to December 2017. The patients were suspected of infection according to the clinical symptoms when they visited the hospital. The samples sources were collected dependent on the suspected tissues of infection including blood, urine, sputum, cervical secretions, pus and others, but not feces. Repeated samples identified in the reviewing process were excluded from our analysis. All the clinical data was retrospectively reviewed anonymously, and the study was approved by the Ethics Committee of Dongyang People's Hospital Ethics Committee and Institutional Review Board.
Species identification and antimicrobial susceptibility test The clinical samples were directly smeared on the Columbia Blood Agar Base (BIO KONT, Wenzhou, China. http://www.bio-kont.cn/) and then incubated at $37{ }^{\circ} \mathrm{C}$ for $18-24 \mathrm{~h}$. When the colonies formed and the suspected $K$. pneumoniae colonies were collected based on the morphology. The species were identified in a clinical laboratory using a Vitek 2 Compact Bacterial Identification and Monitoring System (Biomerieux, France) or a mass spectrometry microbial identification system (Biomerieux, France). Susceptibility to antimicrobial agents commonly used to treat infections was analyzed using the AST-GN13 or AST-N334 (VITEK 2) based on the Clinical and Laboratory Standards Institute (CLSI) breakpoints [16]. The ESBL-positive phenotype was determined using VITEK 2 according to the manufacturer's instructions [17]. The resistance of $K$. pneumoniae to imipenem was further confirmed by the minimum inhibitory concentration test (resistant phenotype: $\geq 1.0 \mu \mathrm{g} / \mathrm{mL}$ ). The reference strains Escherichia coli ATCC 25922, Streptococcus pneumoniae ATCC 49619, Staphylococcus aureus ATCC 29213 Staphylococcus saprophyticus ATCC BAA750 and K. pneumonia ATCC 700324 were used as controls.

\section{The ESBL gene detection of isolates from urine source}

A total of $82 \mathrm{~K}$. pneumoniae isolates were from urine sample from those patients who visited the health care center in 2017. The strains were recovered from $-80{ }^{\circ} \mathrm{C}$ refrigerator and cultured on the Columbia blood agar plates. The colonies formed after an incubation of $24 \mathrm{~h}$ at $37^{\circ} \mathrm{C}$. Then several clones were collected and resuspended in $50 \mu \mathrm{L}$ deionized water. The DNA was extracted from the microorganism by being boiled at $100{ }^{\circ} \mathrm{C}$ for $7 \mathrm{~min}$. After a centrifugation of $10,000 \mathrm{rpm}$ for $5 \mathrm{~min}$, the supernatant containing DNA was collected.

The primers for detecting the TEM and SHV genes were described previously (Additional file 1: Table S1) $[18,19]$. Polymerase chain reaction was carried out in $20 \mu \mathrm{L}$ volumes containing $1 \mu \mathrm{L}$ DNA template, $0.2 \mathrm{mM}$ of dNTPs, $0.25 \mu \mathrm{M}$ of each primer, and $0.1 \mathrm{U}$ of Taq polymerase (Takara, Japan) in $1 \times$ PCR buffer. Amplification of DNA was performed in a thermocycler (Biorad, USA). The cycling parameters were: pre-denaturation ( $95 \mathrm{C}$ for $3 \mathrm{~min}), 35$ thermal cycles of denaturation $\left(95^{\circ} \mathrm{C}\right.$ for $\left.30 \mathrm{~s}\right)$, annealing ( $55 \mathrm{C}$ for $30 \mathrm{~s}$ ), extension ( $72 \mathrm{C}$ for $1 \mathrm{~min}$ ), and final proliferation temperature ( $72 \mathrm{C}$ for $5 \mathrm{~min})$. All the PCR products were analyzed in $1 \%$ agarose gel containing gel staining dye (TRANGENE, China) in Tris-acetate-EDTA buffer, and the gel was photographed using gel documentation system (Bio-Rad, USA). 


\section{Statistical analysis}

The data were managed using WHONET 5.6 version. Differences between resistance rates were analyzed by Chi squared test using SPSS 20.0 version. $P$-values $<0.05$ were considered to indicate statistical significance.

\section{Results}

A total of $5319 \mathrm{~K}$. pneumoniae isolates were obtained from January 2011 to December 2017. The isolation rate of K. pneumoniae in clinical samples ranged from 10.3 to $11.4 \%$, with very small differences by year (Fig. 1). However, the total number of $K$. pneumoniae isolated reached a peak in 2014 and then decreased. The main sample source of K. pneumoniae was sputum (3319, 62.4\%), followed by cervical secretions (514, 9.7\%), urine (495, $9.3 \%)$, blood (300, 5.6\%), pus (294, 5.5\%) and others (397, $7.5 \%)$.

As a known potential drug-resistant pathogen, $K$. pneumoniae has received increasing attention in clinical infections. Thus, in this study, we investigated the resistance of K. pneumoniae to commonly used antimicrobial drugs. The resistance rates varied for the different drugs, from $1.4 \%$ (ertapenem) to $23.1 \%$ (nitrofurantoin) (Table 1). Less than $10 \%$ of the isolates showed resistance to ceftazidime, levofloxacin, ciprofloxacin, cefepime, tobramycin, piperacillin/tazobactam, imipenem, amikacin, cefotetan and ertapenem. In contrast, $10 \%$ to $23 \%$ of $K$. pneumoniae strains showed resistance for nitrofurantoin, cefazolin, sulbactam/ampicillin, ceftriaxone, sulfamethoxazole/trimethoprim, aztreonam and gentamycin. From 2011 to 2016, the rates of resistance to ceftriaxone decreased from 25.6 to $18.5 \%$. However, for ciprofloxacin, the resistance rates increased from 2011 to 2017. The remaining drugs showed only a slight fluctuation in their resistance rates throughout the period of the study.

To investigate the potential difference in drug resistance between different sources of samples, we analyzed the $K$. pneumoniae isolated from urine, sputum, blood and cervical secretions. As shown in Table 2, significant differences in resistance rates to almost all the drugs were observed between the sample sources. Overall, the strains isolated from urine exhibited the highest resistance rates for all the drugs except nitrofurantoin. With regard to some antimicrobial drugs, such as sulfamethoxazole/trimethoprim and ceftazidime, the resistance rates in isolates from urine samples were more than two-fold greater than those isolated from sputum and cervical secretions. In addition, less than $1 \%$ of the $K$. pneumoniae strains isolated from the cervical secretions were resistant to piperacillin/tazobactam, imipenem, amikacin, cefotetan and ertapenem. The distribution of sample sources per year was displayed (Additional file 1: Table S2), with a slight fluctuation across the years.

To investigate the mechanism responsible for the higher resistance rates among $K$. pneumoniae strains isolated from urine samples, we compared the proportion of ESBL-positive strains isolated from the different sources. Overall, ESB-positive strains accounted for $18.7 \%$ (963/5161) of the strains available for ESBLtesting. As expected, the rate of ESBL-positive strains in urine were $27.1 \%$, significantly higher than those in cervical secretions $(20.2 \%, P=0.009)$, blood $(16.5 \%, P<0.001)$ and sputum $(15.2 \%, P<0.001)$. We also analyzed the

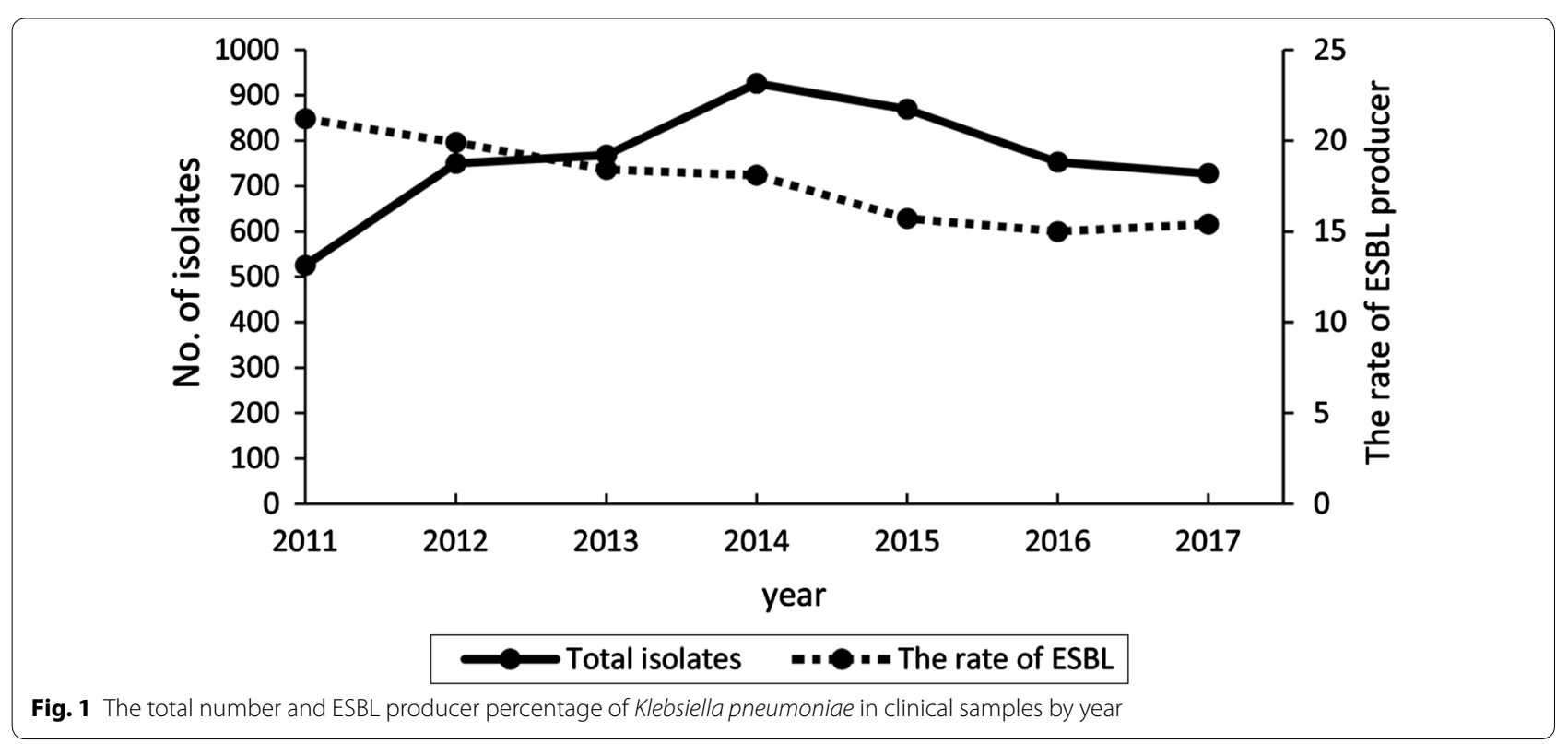


Table 1 The drug resistance rates of Klebsiella pneumoniae by year

\begin{tabular}{|c|c|c|c|c|c|c|c|c|}
\hline Antimicrobial drugs & No. $(\%)^{a}$ & 2011 & 2012 & 2013 & 2014 & 2015 & 2016 & 2017 \\
\hline NIT & $5203(23.1)$ & 13.7 & 20.4 & 24.8 & 29.6 & 33.1 & 12.8 & 20.7 \\
\hline $\mathrm{CZO}$ & $4476(20.3)$ & 25.6 & 21.6 & 20.3 & 20.4 & 18 & 18.5 & - \\
\hline SAM & 5195 (20.6) & 19.8 & 20.5 & 19.8 & 22.4 & 18.4 & 20 & 22.8 \\
\hline CRO & 5204 (18.6) & 23.7 & 21.1 & 18.6 & 19.5 & 15.6 & 17 & 17.2 \\
\hline SXT & $5205(17.3)$ & 17.5 & 19.9 & 19.2 & 18 & 14.5 & 15.4 & 17.2 \\
\hline ATM & $5202(10.4)$ & 10.4 & 11.4 & 10.1 & 11.8 & 9 & 9.3 & 10.6 \\
\hline GEN & $5044(10.4)$ & 12.7 & 11.8 & 10.5 & 12.2 & 8.9 & 9.6 & 8.1 \\
\hline CAZ & $5189(7.5)$ & 8.6 & 9.7 & 6.1 & 7.8 & 7.1 & 7 & 6.4 \\
\hline LVX & $5202(5.5)$ & 3.9 & 7.5 & 5.8 & 6.1 & 3.7 & 5.2 & 5.7 \\
\hline $\mathrm{CIP}$ & $2381(7.0)$ & - & - & - & 4.1 & 5.9 & 7.1 & 8.3 \\
\hline FEP & $5201(4.7)$ & 4.4 & 5.3 & 3.7 & 5.5 & 3.4 & 4.5 & 6.4 \\
\hline TOB & $5202(3.9)$ & 5.3 & 4.7 & 2.9 & 4.8 & 2.7 & 3.9 & 3.7 \\
\hline TZP & $5189(2.5)$ & 2 & 2.7 & 2.1 & 3.4 & 1.5 & 2.4 & 3.3 \\
\hline IPM & $5207(1.8)$ & 1.5 & 1.8 & 1.1 & 2.7 & 0.9 & 2 & 2.2 \\
\hline AMK & $5201(2.0)$ & 3.5 & 2.8 & 1.2 & 2.3 & 0.9 & 1.6 & 2.1 \\
\hline CTT & $5200(2.0)$ & 2.4 & 2.4 & 1.3 & 2.1 & 1.5 & 1.9 & 2.8 \\
\hline ETP & $5141(1.4)$ & 1.8 & 3.4 & 1.8 & 2.9 & 0 & 0 & 0.1 \\
\hline
\end{tabular}

- Data not available

NIT nitrofurantoin, CZO cefazolin, SAM sulbactam/ampicillin, CRO ceftriaxone, SXT sulfamethoxazole/trimethoprim, ATM aztreonam, GEN gentamycin, CAZ ceftazidime, LVX levofloxacin, CIP ciprofloxacin, FEP cefepime, TOB tobramycin, TZP piperacillin/tazobactam, IPM imipenem, AMK amikacin, CTT cefotetan, ETP ertapenem

a The total number of strains profiled with each given antibiotic

Table 2 The antimicrobial drug resistance rates of Klebsiella pneumoniae from different resources

\begin{tabular}{|c|c|c|c|c|c|}
\hline Antimicrobial drug & Blood, 300 & Sputum, 3319 & Genital secretion, 514 & Urine, 495 & $P$ value \\
\hline NIT & 35.2 & 20.9 & 20.7 & 30.7 & $<0.001$ \\
\hline CZO & 23.6 & 19.5 & 22.6 & 35.1 & $<0.001$ \\
\hline SAM & 20.7 & 18 & 21.1 & 32.6 & $<0.001$ \\
\hline CRO & 18.6 & 16.6 & 19.3 & 29.1 & $<0.001$ \\
\hline SXT & 16 & 14 & 24 & 28.3 & $<0.001$ \\
\hline ATM & 11.3 & 9.2 & 9.4 & 18.8 & $<0.001$ \\
\hline GEN & 10.1 & 8.8 & 11.9 & 16.9 & $<0.001$ \\
\hline CAZ & 9 & 6.5 & 5.9 & 14.2 & $<0.001$ \\
\hline LVX & 7.6 & 3.6 & 4.7 & 14.8 & $<0.001$ \\
\hline CIP & 6.1 & 5.3 & 8.4 & 14.5 & $<0.001$ \\
\hline FEP & 5.5 & 4.2 & 1.8 & 10.7 & $<0.001$ \\
\hline TOB & 3.2 & 3.3 & 2.7 & 8.8 & $<0.001$ \\
\hline TZP & 3.2 & 2.5 & 0.2 & 4.7 & $<0.001$ \\
\hline IPM & 2.9 & 2 & 0 & 3.6 & $<0.001$ \\
\hline AMK & 2.3 & 1.7 & 0.2 & 4.7 & $<0.001$ \\
\hline СTT & 1.7 & 2 & 0.2 & 4.3 & $<0.001$ \\
\hline ETP & 0.9 & 1.5 & 0.2 & 2.7 & $<0.001$ \\
\hline
\end{tabular}

NIT nitrofurantoin, CZO cefazolin, SAM sulbactam/ampicillin, CRO ceftriaxone, SXT sulfamethoxazole/trimethoprim, ATM aztreonam, GEN gentamycin, CAZ ceftazidime, LVX levofloxacin, CIP ciprofloxacin, FEP cefepime, TOB tobramycin, TZP piperacillin/tazobactam, IPM imipenem, AMK amikacin, CTT cefotetan, ETP ertapenem

association between ESBL expression and drug resistance by comparing the resistance rates between the ESBL-positive and ESBL-negative strains (Table 3).
Among the ESBL-negative strains, more than $90 \%$ were susceptible to all the antimicrobial drugs investigated, except nitrofurantoin. Compared to the ESBL-negative 
Table 3 The drug resistance rates between ESBL (-) and ESBL (+) Klebsiella pneumoniae

\begin{tabular}{lllr}
\hline Antimicrobial drugs & $\begin{array}{l}\text { ESBL (-), } \\
\mathbf{n = 4 1 9 8}\end{array}$ & $\begin{array}{l}\text { ESBL }(+), \\
\mathbf{n = 9 6 3}\end{array}$ & $P$ value \\
\hline NIT & 19.8 & 39.3 & $<0.001$ \\
CZO & 4.7 & 95.8 & $<0.001$ \\
SAM & 8 & 80.3 & $<0.001$ \\
CRO & 2.6 & 93.3 & $<0.001$ \\
SXT & 8.2 & 60.7 & $<0.001$ \\
ATM & 2.2 & 49.1 & $<0.001$ \\
GEN & 3.4 & 44.2 & $<0.001$ \\
CAZ & 2.5 & 30 & $<0.001$ \\
LVX & 2.7 & 17.9 & $<0.001$ \\
CIP & 2.7 & 29.3 & $<0.001$ \\
FEP & 1.7 & 19 & $<0.001$ \\
TOB & 1.8 & 13.4 & $<0.001$ \\
TZP & 2.2 & 3.8 & 0.005 \\
IPM & 1.9 & 1.6 & 0.491 \\
AMK & 1.4 & 4.4 & $<0.001$ \\
CTT & 1.8 & 2.1 & 0.554 \\
ETP & 1.3 & 2.6 & 0.005 \\
\hline
\end{tabular}

NIT nitrofurantoin, CZO cefazolin, SAM sulbactam/ampicillin, CRO ceftriaxone, SXT sulfamethoxazole/trimethoprim, ATM aztreonam, GEN gentamycin, CAZ ceftazidime, $L V X$ levofloxacin, CIP ciprofloxacin, FEP cefepime, TOB tobramycin, TZP piperacillin/tazobactam, IPM imipenem, AMK amikacin, CTT cefotetan, ETP ertapenem

K. pneumoniae strains, the ESBL-positive strains showed higher resistance rates to all of antimicrobial drugs except imipenem and cefotetan. For example, less than $10 \%$ of the ESBL-negative strains showed resistance to cefazolin, sulfamethoxazole, ceftriaxone and sulbactam/ampicillin, whereas more than $60 \%$ of ESBL-positive strains showed resistance to these drugs.

The most common ESBL gene was SHV gene (28\%, $23 / 82)$, following by TEM $(14.6 \%, 12 / 82)$. The percentage of SHV genes in ESBL producer $(14.3 \%, 3 / 21)$ was lower than that in non-ESBL producer $(32.8 \%, 20 / 61)$. The percentages of TEM genes in ESBL producer $(19.4 \%, 4 / 21)$ strains were higher than in non-ESBL producer $(13.1 \%$, 8/61) strains.

\section{Discussion}

Along with a high prevalence, K. pneumoniae is a known pathogen antibiotic resistant pathogen. As expected, there was a high variability among the isolates investigated in the prevalence of resistance to the different antibiotics, which could be explained by distinct resistance mechanisms [3], including the expression of ESBLs in $K$. pneumoniae strains. The decreased number of isolates and the rate of ESBL producer in this study may be due to more acknowledge of the K. pneumoniae infection, which could be diagnosed by combination with other examination methods.

In general, the ESBL-positive strains were resistant to advanced generation cephalosporins [7], with resistance rates to ceftriaxone, ceftazidime and cefepime found to be more than tenfold higher than those of the ESBLnegative strains (Table 3). In addition, the ESBL-positive strains showed higher resistance rates to aminoglycosides (gentamycin, amikacin and tobramycin), fluoroquinolones (ciprofloxacin) and other agents (Table 3). The crossresistance in ESBL-positive strains may be due to the plasmids carried by the pathogens, resulting in the emergence and spread of multi-drug resistant strains among the individuals $[3,7,8]$. Thus, investigations of the ESBLpositive strains are required to elucidate the mechanism underlying multi-drug resistance and to identify potential targets of new antibiotics.

We found that the resistances rates varied significantly among the $K$. pneumoniae strains isolated the different sample sources. The highest resistance rates to most of the drugs tested were identified in strains isolated from urine, which may be due to the highest rate of EBSL-positive strains isolated from this source. Indeed, there is a demographic variety of ESBL-positive rates in K. pneumoniae strain isolated from urine. At $27.1 \%$, the rate of ESBL-positive strains in urine identified in our study is higher than that (18.4\%) reported in Nepal [14], but lower than that (47.5\%) in Saudi Arabia [15]. Although there was a significant increase in the nitrofurantoin resistance rates of ESBL-positive strains isolated during the study period, the highest resistance rates were detected among the strains isolated from blood, which exhibited a lower ESBL-positive rate. Thus, caution should be exercised in the use of nitrofurantoin to treat $K$. pneumoniae infection of the blood due to the possibility of resistance leading to increased mortality [20-22]. A survey conducted in the United States also revealed significant differences in drug resistance rates among isolates from different sample sources, with higher resistance rates in K. pneumoniae strains isolated from the lower respiratory tract than those from urine [4]. In contrast to our findings, the same study showed that the resistance rates to all the antibiotic agents were the lowest in the urine sample isolates.

Although the distribution of ESBL genes in strains from urine source could not explain the higher resistance, it could indicate that the ESBL genes may be served as molecular detection markers, instead of contributing to the ESBL phenotype [23]. In another way, the higher resistance of strains from the urine may be speculated by the mechanism in pathogenicity in urinary tract system as reported previously [24]. The isolates should overcome several obstacles including the flow of urine and low $\mathrm{pH}$, and finally colonized in the urinary tract. This ability may 
result from unique component such as type 1 fimbriae, which was expressed in strains from urinary tract but not from gastrointestinal tract or lungs [25]. Furthermore, fimbriae expression would be of great importance in biofilm formation and the biofilm was a structure where the strains owned more resistance to chemicals [26].

Comparisons of the resistance rates of K. pneumoniae strains isolated from different sample sources are rare; therefore, a comprehensive survey of the potential differences among the strains from different sample sources is urgently required. Moreover, the differences in drug resistance among clinical samples from various sources indicates that optimal drug strategies should be considered based on the site of K. pneumoniae infection.

\section{Conclusions}

In conclusion, our investigation of the resistance rates of K. pneumoniae strains from a primary hospital revealed that the resistance rates of strains in urine samples to most antibiotics are higher than those in blood, sputum and cervical secretion from females. This is associated with a corresponding increase in number of ESBL-positive strains. Therefore, the contribution of ESBL expression to antimicrobial resistance or cross-resistance warrants further investigation.

\section{Supplementary information}

Supplementary information accompanies this paper at https://doi. org/10.1186/s12941-019-0335-8.

Additional file 1. Additional tables.

\section{Abbreviation}

ESBL: extended-spectrum $\beta$-lactamases.

\section{Acknowledgements}

None.

\section{Authors' contributions}

PX conducted the design and was major contributor in writing the manuscript. LG and ZS collected the data and performed the statistical analysis. ZY, WS and SY performed the anti-microbial susceptibility assay and data analysis. All authors read and approved the final manuscript.

\section{Funding}

No funding was received.

\section{Availability of data and materials}

All data generated or analyzed during this study are included in this published article.

\section{Ethics approval and consent to participate}

Not applicable.

\section{Consent for publication}

Not applicable.

\section{Competing interests}

The authors declare that they have no competing interests.

\section{Author details}

${ }^{1}$ Department of Clinical Laboratory, Affiliated Dongyang Hospital of Wenzhou Medical University, Dongyang, Zhejiang, China. ${ }^{2}$ Department of Biomedical Sciences Laboratory, Affiliated Dongyang Hospital of Wenzhou Medical University, No. 60 Wuningxi Road, Dongyang, Zhejiang, China.

Received: 13 April 2019 Accepted: 29 October 2019

Published online: 09 November 2019

\section{References}

1. Boonsarngsuk V, Thungtitigul P, Suwatanapongched T. Chronic Klebsiella pneumoniae: a rare manifestation of Klebsiella pneumoniae. J Thorac Dis. 2015;7:1661-4

2. Siu LK, Yeh KM, Lin JC, Fung CP, Chang FY. Klebsiella pneumoniae liver abscess: a new invasive syndrome. Lancet Infect Dis. 2012;12:881-7.

3. Navon-Venezia S, Kondratyeva K, Carattoli A. Klebsiella pneumoniae: a major worldwide source and shuttle for antibiotic resistance. FEMS Microbiol Rev. 2017:41:252-75.

4. Sanchez GV, Master RN, Clark RB, Fyyaz M, Duvvuri P, Ekta G, Bordon J. Klebsiella pneumoniae antimicrobial drug resistance, United States, 1998-2010. Emerg Infect Dis. 2013;19:133-6.

5. De Laveleye M, Huang TD, Bogaerts P, Berhin C, Bauraing C, Sacre P, Noel A, Glupczynski Y, multicenter study g. Increasing incidence of carbapenemase-producing Escherichia coli and Klebsiella pneumoniae in Belgian hospitals. Eur J Clin Microbiol Infect Dis. 2017;36:139-46.

6. Campos AC, Albiero J, Ecker AB, Kuroda CM, Meirelles LE, Polato A Tognim MC, Wingeter MA, Teixeira JJ. Outbreak of Klebsiella pneumoniae carbapenemase-producing K. pneumoniae: a systematic review. Am J Infect Control. 2016:44:1374-80.

7. Padmini N, Ajilda AAK, Sivakumar N, Selvakumar G. Extended spectrum beta-lactamase producing Escherichia coli and Klebsiella pneumoniae: critical tools for antibiotic resistance pattern. J Basic Microbiol. 2017:57:460-70.

8. Ma L, Lin CJ, Chen JH, Fung CP, Chang FY, Lai YK, Lin JC, Siu LK, Taiwan Surveillance of Antimicrobial Resistance P. Widespread dissemination of aminoglycoside resistance genes arm $A$ and $r m t B$ in Klebsiella pneumoniae isolates in Taiwan producing CTX-M-type extended-spectrum betalactamases. Antimicrob Agents Chemother. 2009;53:104-11.

9. Liang C, Xing B, Yang X, Fu Y, Feng Y, Zhang Y. Molecular epidemiology of aminoglycosides resistance on Klebsiella pneumoniae in a hospital in China. Int J Clin Exp Med. 2015;8:1381-5.

10. Yang Q, Zhang H, Wang Y, Xu Z, Zhang G, Chen X, Xu Y, Cao B, Kong $\mathrm{H}, \mathrm{Ni} Y$, et al. Antimicrobial susceptibilities of aerobic and facultative gram-negative bacilli isolated from Chinese patients with urinary tract infections between 2010 and 2014. BMC Infect Dis. 2017;17:192.

11. He LY, Wang YJ, Li JM. Clinical features and antimicrobial resistance of community-acquired pneumonia caused by Klebsiella pneumoniae in infants. Zhongguo Dang Dai Er Ke Za Zhi. 2012;14:827-9.

12. Li Y, Cao X, Ge H, Jiang Y, Zhou H, Zheng W. Targeted surveillance of nosocomial infection in intensive care units of 176 hospitals in Jiangsu province. China. J Hosp Infect. 2018;99:36-41.

13. Liu C, Shi J, Guo J. High prevalence of hypervirulent Klebsiella pneumoniae infection in the genetic background of elderly patients in two teaching hospitals in China. Infect Drug Resist. 2018;11:1031-41.

14. Ma Y, Bao C, Liu J, Hao X, Cao J, Ye L, Yang J. Microbiological characterisation of Klebsiella pneumoniae isolates causing bloodstream infections from five tertiary hospitals in Beijing, China. J Glob Antimicrob Resist. 2018;12:162-6.

15. Zhang Y, Zhao C, Wang Q, Wang X, Chen H, Li H, Zhang F, Li S, Wang R, Wang $\mathrm{H}$. High prevalence of hypervirulent Klebsiella pneumoniae Infection in China: geographic distribution, clinical characteristics, and antimicrobial resistance. Antimicrob Agents Chemother. 2016;60:6115-20.

16. Clinical and Laboratory Standards Institute. Performance standards for antimicrobial susceptibility testing, 20th ed. Document M100-S23. Wayne, PA: Clinical and Laboratory Standards Institute; 2013.

17. Clinical and Laboratory Standards Institute. Methods for dilution antimicrobial susceptibility tests for bacteria that grow aerobically, Approved standard: 8th ed. Document M7-A8. Wayne, PA: Clinical and Laboratory Standards Institute; 2009. 
18. Yu Y, Ji S, Chen Y, Zhou W, Wei Z, Li L, Ma Y. Resistance of strains producing extended-spectrum beta-lactamases and genotype distribution in China. J Infect. 2007;54:53-7.

19. Zhou HW, Zhang T, Ma JH, Fang Y, Wang HY, Huang ZX, Wang Y, Wu C, Chen GX. Occurrence of plasmid- and chromosome-carried mcr-1 in waterborne Enterobacteriaceae in China. Antimicrob Agents Chemother 2017. https://doi.org/10.1128/AAC.00017-17.

20. Gentry CA, Williams RJ 2nd. Trends in susceptibility rates and extendedspectrum beta-lactamase production of Klebsiella pneumoniae in bloodstream infections across the United States veterans affairs healthcare system. Microb Drug Resist. 2015;21:590-9.

21. Pau CK, Ma FF, Ip M, You JH. Characteristics and outcomes of Klebsiella pneumoniae bacteraemia in Hong Kong. Infect Dis. 2015;47:283-8.

22. Brady M, Cunney R, Murchan S, Oza A, Burns K. Klebsiella pneumoniae bloodstream infection, antimicrobial resistance and consumption trends in Ireland: 2008 to 2013. Eur J Clin Microbiol Infect Dis. 2016:35:1777-85.
23. Bajpai T, Pandey M, Varma M, Bhatambare GS. Prevalence of TEM, SHV and CTX-M beta-lactamase genes in the urinary isolates of a tertiary care hospital. Avicenna J Med. 2017;7:12-6.

24. Paczosa MK, Mecsas J. Klebsiella pneumoniae: going on the offense with a strong defense. Microbiol Mol Biol Rev. 2016;80:629-61.

25. Struve C, Bojer M, Krogfelt KA. Characterization of Klebsiella pneumoniae type 1 fimbriae by detection of phase variation during colonization and infection and impact on virulence. Infect Immun. 2008;76:4055-65.

26. Davies D. Understanding biofilm resistance to antibacterial agents. Nat Rev Drug Discov. 2003;2:114-22.

\section{Publisher's Note}

Springer Nature remains neutral with regard to jurisdictional claims in published maps and institutional affiliations.
Ready to submit your research? Choose BMC and benefit from:

- fast, convenient online submission

- thorough peer review by experienced researchers in your field

- rapid publication on acceptance

- support for research data, including large and complex data types

- gold Open Access which fosters wider collaboration and increased citations

- maximum visibility for your research: over $100 \mathrm{M}$ website views per year

At BMC, research is always in progress.

Learn more biomedcentral.com/submissions 